\title{
Artificial neural network analysis of lipase-catalyzed synthesis of sugar alcohol ester.
}

\begin{abstract}
Artificial neural network (ANN) analysis of immobilized Candida antarctica lipase Bcatalyzed esterification of palmitic acid with xylitol was carried out. Temperature, time, amount of enzyme, amount of molecular sieve, substrate molar ratio and volume of solvent were the six important parameters used as the inputs of the network trained by LevenbergMarquardt (LM) algorithm. After evaluating different ANN configurations, the best network was found to be consisted of two hidden layers with six and seven neurons in the first and second layers respectively, using a hyperbolic tangent sigmoid transfer function. The coefficient of determination (R2) and mean square error (MSE) values between the actual and predicted responses were 1 and $1.5025 \mathrm{e}-24$ for the training and 0.97239 and 0.03259 for the testing datasets. The results indicate the good generalization performance of the neural network model and its capability to predict the conversion of the substrates.
\end{abstract}

Keyword: Artificial neural network; Xylitol; Sugar alcohol; Lipase; Esterification. 